\title{
Improved Chirp Scaling Algorithm Based on Fractional Fourier Trans- form and Motion Compensation
}

\author{
Tan Gewei", Pan Guangwu and Lin Wei
}

School of Information Science and Engineering, Huaqiao University, Xiamen, Fujian, P.R. China

\begin{abstract}
Fractional Fourier transform (FrFT) is a new tool of time-frequency analysis, and a good one to compress chirp signal and to process non-stationary motion error. Aiming at the problem of the conventional SAR imaging algorithm based on FFT and two-step motion compensation, that the effect of non-stationary error elimination is not obvious and the improvement of resolution is restricted, an improved Chirp Scaling imaging algorithm based on FrFT and motion compensation is put forward in this paper, which is expected to eliminate the influence of range motion error, so as to obtain high quality SAR images. Simulation results and imaging results of real SAR data show that the proposed algorithm can eliminate the influence of range motion error effectively (the real SAR data provided by Institute of Electronics, Chinese Academy of Sciences).
\end{abstract}

Keywords: Fractional Fourier transform, Non-stationary motion error, Motion compensation, CS algorithm, High resolution.

\section{INTRODUCTION}

Motion error is a crucial factor to limit airborne SAR resolution improvement. It is necessary to take measures to compensate the motion error caused by air turbulence to obtain high quality SAR images [1-3]. The two-dimensional spatial-variant property of SAR motion error is a challenge to motion compensation technique. Two-step motion compensation algorithm can eliminate the influence of range motion errors, but the effect of compensation is not obvious when motion errors are non-stationary.

Fractional Fourier transform (FrFT) is proposed by V. Namias in 1980 [4], which is a new time-frequency analysis tool. Compared to Fourier transform, FrFT has incomparable superiority in processing non-stationary signals. Chirp signal can be well focused after fractional Fourier transform with the specific rotation angle which provides a possibility to achieve high resolution and high accuracy in SAR imaging. Especially when signal and interference source are coupled to each other, good separation effect in fractional domain can be obtained [5-7].

There have been literatures putting forward SAR imaging algorithm combining with FrFT. Literature [8, 9] proposed an improving CS imaging algorithm based on FrFT. Literature 10 and 11 also proposed a combination of FrFT and RD imaging algorithm. These research results show that FrFT can contribute to the improvement of SAR resolution.

*Address correspondence to this author at the School of Information Engineering, Huaqiao University, Xiamen, Fujian, 361021, P.R. China;

Tel: 13799763568; E-mail: tangewei70@163.com
In order to eliminate the influence of motion error more effectively, in the paper, FrFT is combined with SAR imaging algorithm and motion compensation technology, through utilizing the superiority of FrFT in processing chirp signal and non-stationary signal, so as to obtain high quality SAR images.

\section{FRACTIONAL FOURIER TRANSFORM}

Fractional Fourier transform is the general form of Fourier transform, and its transform kernel is

$$
K_{p}(t, u)=\left\{\begin{array}{lc}
\sqrt{\frac{1-j \cot \alpha}{2 \pi}} \exp \left\{j \pi \left(t^{2}+u^{2}\right.\right. & \cot \alpha-2 u t \csc \alpha)\} \alpha \notin n \pi \\
\delta(t-u) & \alpha \in n \cdot 2 \pi \\
\delta(t+u) & \alpha+\pi \in n \cdot 2 \pi
\end{array}\right.
$$

where $\alpha=p \cdot \frac{\pi}{2}, \quad p$ is the order of FrFT, when $\alpha=\frac{\pi}{2}$, the FrFT becomes the Fourier transform.

Fractional Fourier transform and its inverse transform for signal $x(t)$ is defined as:

$$
\begin{aligned}
& X(u)=F_{p}[x(t)](u)=\int_{-\infty}^{\infty} x(t) K_{p}(t, u) d t \\
& x(t)=F_{-p}[X(u)](t)=\int_{-\infty}^{\infty} X(u) K_{-p}(t, u) d u
\end{aligned}
$$

Fractional Fourier transform for chirp signal $x(t)=e^{j 2 \pi k t^{2}}$ is as follows:

$$
X(u)=\int_{-\infty}^{\infty} x(t) K_{p}(t, u) d t=A e^{j \pi u^{2} \cot \alpha} \int_{-\infty}^{\infty} e^{j 2 \pi k t^{2}} e^{j \pi t^{2} \cot \alpha-j 2 \pi u t \csc \alpha} d t
$$


where $A=\sqrt{\frac{1-j \cot \alpha}{2 \pi}}$.

When $\cot \alpha=-2 k, p=-\frac{2}{\pi} \operatorname{arccot}(2 k)=p_{\text {opt }}$, which is the optimal order of FrFT. Thus,

$$
\begin{aligned}
X(u) & =A e^{-j 2 \pi k u^{2}} \int_{-\infty}^{\infty} e^{-j 2 \pi u t \csc \alpha} d t \\
& =A e^{-j 2 \pi k u^{2}} \delta(2 \pi \csc \alpha u)=\frac{A}{\csc \alpha} \delta(u)=C \delta(u)
\end{aligned}
$$

So the optimal-order fractional Fourier transform of a chirp signal is an impulse signal.

FrFT has the following time-shift and frequency-shift properties,

$$
\begin{gathered}
F_{p}\left[s\left(t+\tau_{0}\right)\right](u)=\mathrm{e}^{j \pi \tau_{0}^{2} \sin \alpha \cos \alpha+j 2 \pi \tau_{0} u \sin \alpha} F_{p}[s(t)]\left(u+\tau_{0} \cos \alpha\right) \\
F_{p}\left[s(t) e^{j 2 \pi \rho t}\right](u)=\mathrm{e}^{-j \pi p^{2} \sin \alpha \cos \alpha+j 2 \pi \rho u \cos \alpha} F_{p}[s(t)](u-\rho \sin \alpha) \\
F_{p}\left[e^{j 2 \pi \rho t} s\left(t+\tau_{0}\right)\right](u)=F_{p}[s(t)]\left(u+\tau_{0} \cos \alpha-\rho \sin \alpha\right) \\
\cdot \exp \left\{j \pi\left(\begin{array}{l}
\tau_{0}^{2} \sin \alpha \cos \alpha+2 \tau_{0} u \sin \alpha+2 \rho u \cos \alpha \\
-\rho^{2} \sin \alpha \cos \alpha-2 \rho \tau_{0} \sin ^{2} \alpha
\end{array}\right)\right\}
\end{gathered}
$$

According to frequency-shift property, there is,

$F_{p_{\text {opt }}}\left[\mathrm{e}^{j 2 \pi\left(k t^{2}+v t\right)}\right](u)=C \delta(u-v \sin \alpha)$

if the chirp signal is a discrete sequence, then the optimal order is $p_{\text {opt }}=-\frac{2}{\pi} \operatorname{arccot}\left(\frac{2 k}{f_{s}^{2} / N}\right)$.

Just using the optimal-order fractional Fourier transform, chirp signals will be well focused, where there is no need of additional matching compression, thereby improving processing efficiency.

\section{MOTION ERROR MODEL AND SAR ECHO SIG-} NAL

Motion error in range direction is divided into rangeindependent and range-dependent motion errors [12]. The part unrelated to range slant is called $\Delta r_{m}\left(t, r_{m}\right)$, and the part that is dependent on the change of range slant is called $\Delta r_{v}\left(t, r_{0}\right)$. Range motion error refers to the difference of the nearest distance from one target to real track and the nearest distance from the target to the nominal track [13].

$$
\Delta r\left(t, r_{0}\right)=\Delta r_{m}\left(t, r_{m}\right)+\Delta r_{v}\left(t, r_{0}\right)
$$

where $r_{m}$ is the nearest slant range of swath band center, $w_{r}$ is the width of swath band, $r_{0}$ is the nearest slant range of the target $P$ under nominal track.

Suppose that SAR transmit chirps to an observed scene, their echoes after the demodulation are

$$
\begin{gathered}
s d\left(t, \tau ; r_{0}\right)=\sigma\left(x_{0}, r_{0}\right) \cdot \operatorname{rect}\left(\frac{\tau-2 R(t) / c}{T_{p}}\right) \exp \left\{-j \pi k\left(\tau-\frac{2 R(t)}{c}\right)^{2}\right\} \\
\cdot \operatorname{rect}\left(\frac{t-t_{0}}{T_{s}}\right) \exp \left\{-j \frac{4 \pi R(t)}{\lambda}\right\}
\end{gathered}
$$

where $\tau$ is fast time in the slant range direction, $t$ is slow time along the radar flight path, $c$ and $\lambda$ are speed of light and radar wavelength respectively, and $k$ is the chirp rate. rect ( ) is the rectangle function, in which $T_{p}$ and $T_{s}$ are the pulse duration and synthetic aperture time respectively. $R(t)$ is the instant range from the radar to a point target in the observed scene, which includes motion error.

$$
R(t)=R_{0}(t)+\Delta r\left(t, r_{0}\right)=R_{0}(t)+\Delta r_{m}\left(t, r_{m}\right)+\Delta r_{v}\left(t, r_{0}\right)
$$

where $R_{0}(t)=\sqrt{r_{0}^{2}+v^{2}\left(t-t_{0}\right)^{2}}$ is instant range from radar to the point target under nominal track.

Ignoring azimuth motion errors, range-Fourier transform is first performed by the stationary phase principle, the result is:

$$
\begin{gathered}
s D\left(t, f_{r} ; r_{0}\right)=C_{1} \cdot \sigma\left(x_{0}, r_{0}\right) \cdot r e c t\left(\frac{f_{r}}{B_{r}}\right) \cdot r e c t\left(\frac{t-t_{0}}{T_{s}}\right) \cdot \exp \left\{j \pi \frac{f_{r}^{2}}{k}\right\} \\
\cdot \exp \left\{-j \frac{4 \pi \Delta r_{m}\left(t, r_{m}\right)}{c} f_{r}\right\} \cdot \exp \left\{-j \frac{4 \pi \Delta r_{v}\left(t, r_{0}\right)}{c} f_{r}\right\} \\
\cdot \exp \left\{-j \frac{4 \pi R_{0}}{\lambda}\right\} \cdot \exp \left\{-j \frac{4 \pi \Delta r_{m}\left(t, r_{m}\right)}{\lambda}\right\} \cdot \exp \left\{-j \frac{4 \pi \Delta r_{v}\left(t, r_{0}\right)}{\lambda}\right\}
\end{gathered}
$$

where $f_{r}$ is range frequency.

Range-independent motion errors can cause the echo envelope delay errors (the second exponential item) and azimuth phase errors (the fifth exponential item), which should be compensated along with range compress. Rangedependent motion error (the third and sixth exponential item) is related to slant range. In real SAR data, all point targets are spread out in range direction and azimuth direction, so compensation to such error must be implemented when range compression and the range cell migration correction (RCMC) are completed and azimuth compression is unfinished.

\section{IMPROVED CS ALGORITHM BASED ON FRAC- TIONAL FOURIER TRANSFORM AND MOTION COMPENSATION}

Processing flow of the improved CS algorithm based on FrFT and motion compensation is shown in Fig. (1).

Firstly, perform range-Fourier transform for raw data and the result is formula 10 , which when multiplying with $H_{\text {mocol } 1}$ can complete first-order motion compensation, and the phase compensation function is:

$$
H_{\text {mocol }}\left(t, f_{r} ; r_{m}\right)=\exp \left\{j \frac{4 \pi}{c}\left(f_{r}+f_{c}\right) \Delta r_{m}\left(t, r_{m}\right)\right\}
$$




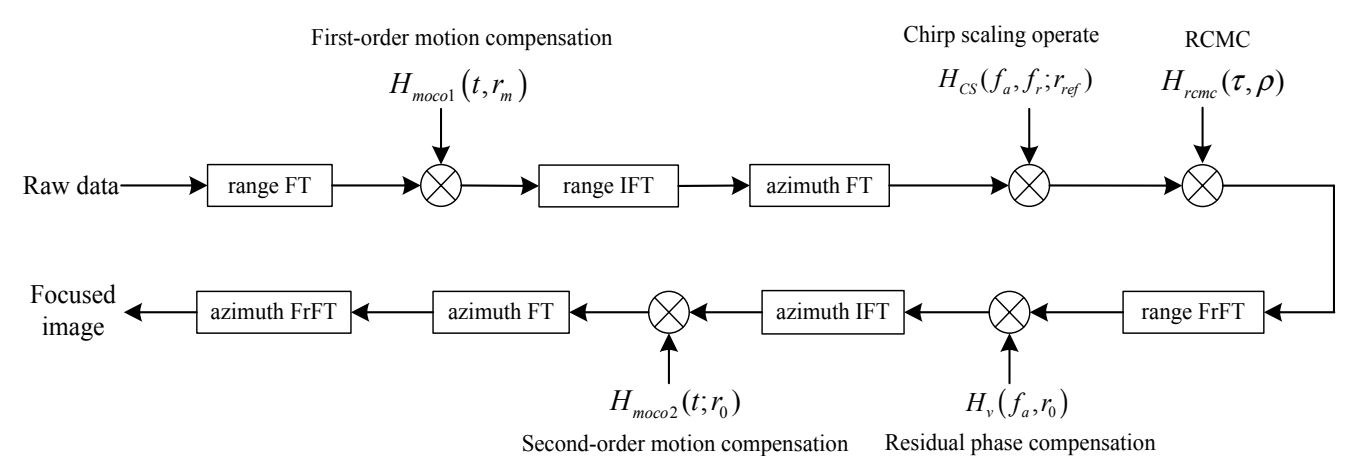

Fig. (1). Processing flow of the improved CS algorithm based on FrFT and motion compensation.

So range-independent motion errors are removed from the raw data. After first-order motion compensation, the echo signal is,

$$
\begin{aligned}
& s d\left(t, \tau ; r_{0}\right)=\sigma\left(x_{0}, r_{0}\right) \cdot \operatorname{rect}\left(\frac{t-t_{0}}{T_{s}}\right) \cdot \exp \left\{-j \frac{4 \pi\left(R_{0}+\Delta r_{v}\right)}{\lambda}\right\} \\
& \cdot \operatorname{rect}\left(\frac{\tau-2\left(R_{0}+\Delta r_{v}\right) / c}{T_{p}}\right) \cdot \exp \left\{-j \pi k\left(\tau-\frac{2\left(R_{0}+\Delta r_{v}\right)}{c}\right)^{2}\right\}
\end{aligned}
$$
is

Then performing azimuth-Fourier transform, the result

$$
\begin{array}{r}
S d\left(f_{a}, \tau ; r_{0}\right)=C_{2} \cdot \sigma\left(x_{0}, r_{0}\right) \cdot \operatorname{rect}\left(\frac{f_{a}}{B_{d}}\right) \exp \left\{-j \frac{4 \pi R\left(f_{a} ; r_{0}\right)}{\lambda}\right\} \\
\cdot \operatorname{rect}\left(\frac{\tau-2 R\left(f_{a} ; r_{0}\right) / c}{T_{p}}\right) \exp \left\{-j \pi k_{m}\left(\tau-\frac{2 R\left(f_{a} ; r_{0}\right)}{c}\right)^{2}\right\}
\end{array}
$$

where $f_{a}$ is Doppler frequency, $C_{2}$ is a complex constant,

$$
\begin{aligned}
& R\left(f_{a} ; r_{0}\right)=\frac{r_{0}+\Delta r_{v}}{\gamma\left(f_{a}\right)}=\left(r_{0}+\Delta r_{v}\right)\left[1+C_{s}\left(f_{a}\right)\right], \gamma\left(f_{a}\right)=\sqrt{1-\left(\frac{\lambda f_{a}}{2 v}\right)^{2}}, \\
& k_{m}=\frac{1}{1 / k+1 / K_{S R C}}, K_{S R C}=\frac{\gamma^{3}\left(f_{a}\right)}{1-\gamma^{2}\left(f_{a}\right)} \cdot \frac{c^{2}}{2 \lambda r_{0}} .
\end{aligned}
$$

Next CS operation is performed, the corresponding processing function is:

$H_{C S}\left(f_{a}, \tau ; r_{r e f}\right)=\exp \left\{-j \pi k_{m}\left(f_{a} ; r_{r e f}\right) C_{s}\left(f_{a}\right)\left(\tau-\tau_{r e f}\left(f_{a}\right)\right)^{2}\right\}$

where $r_{r e f}$ is reference range, $\tau_{r e f}=\frac{2 r_{r e f}}{c}\left[1+C_{s}\left(f_{a}\right)\right]$.

Then multiplying formula 13 with formula 12 , the result after CS operation is:

$$
\begin{aligned}
S d_{C S}\left(f_{a}, \tau ; r_{0}\right)=S d\left(f_{a}, \tau ; r_{0}\right) \cdot H_{C S}\left(f_{a}, \tau ; r_{r e f}\right) \\
=C_{2} \cdot \sigma\left(x_{0}, r_{0}\right) \cdot \operatorname{rect}\left(\frac{f_{a}}{B_{d}}\right) \exp \left\{-j \frac{4 \pi\left(r_{0}+\Delta r_{v}\right)}{\lambda} \gamma\left(f_{a}\right)\right\} \\
\cdot \exp \left\{-j \Theta\left(f_{a} ; r_{0}\right)\right\} \operatorname{rect}\left[\frac{1}{T_{p}}\left(\tau-\frac{2}{c}\left(r_{0}+r_{r e f} C_{s}\left(f_{a}\right)\right)\right)\right] \\
\quad \cdot \exp \left\{-j \pi k_{m}\left[1+C_{s}\left(f_{a}\right)\right]\left[\tau-\frac{2}{c}\left(r_{0}+r_{r e f} C_{s}\left(f_{a}\right)\right)\right]^{2}\right\}
\end{aligned}
$$

where $\Theta$ is residual phase caused by CS operation,

$$
\Theta\left(f_{a} ; r_{0}\right)=\frac{4 \pi}{c^{2}} k_{m}\left(f_{a}, r_{r e f}\right) C_{s}\left(f_{a}\right)\left[1+C_{s}\left(f_{a}\right)\right]\left(r_{0}-r_{r e f}\right)^{2}
$$

From formula 14, it is known that the migration track of point target of different range is same as that of reference point. So bulk RCMC can be performed.

Using the focusing properties of FrFT in processing chirp signal, and its time-shift and frequency-shift properties, the range compression and RCMC are shown as follows. Formula 14 is rewritten as a chirp signal with time-shift,

$$
\begin{aligned}
& S d_{C S}\left(f_{a}, \tau ; r_{0}\right)=s_{d}\left(f_{a}, \tau+\tau_{0} ; r_{0}\right) \cdot \exp \left\{-j \frac{4 \pi\left(r_{0}+\Delta r_{v}\right)}{\lambda} \gamma\left(f_{a}\right)-j \Theta\right\} \\
& s_{d}\left(f_{a}, \tau+\tau_{0} ; r_{0}\right)=C_{2} \cdot \sigma \cdot \operatorname{rect}\left[\frac{1}{T_{p}}\left(\tau+\tau_{0}\right)\right] \\
& \exp \left\{-j \pi k_{m}\left[1+C_{s}\left(f_{a}\right)\right]\left(\tau+\tau_{0}\right)^{2}\right\}
\end{aligned}
$$

where $\tau_{0}=-\frac{2}{c}\left(r_{0}+r_{r e f} C_{s}\left(f_{a}\right)\right)$, and the RCM term is $-2 r_{r e f} C_{s} / c$

According to formula 6, phase compensation function $H_{r c m c}(\tau, \rho)=e^{j \pi \rho \tau}$ is needed to offset the influence of RCM term. Then range-Fractional Fourier transform of the optimal order $p_{\text {opt }}=-\frac{2}{\pi} \operatorname{arccot}\left(2 k_{m}\left(1+C_{s}\right)\right)$ is performed, the result is:

$$
\begin{aligned}
& F_{p}\left[S d_{C S}\left(f_{a}, \tau+\tau_{0} ; r_{0}\right) H_{r c m c}\left(f_{a}, \tau\right)\right](u)= \\
& F_{p}\left[s_{d}\left(f_{a}, \tau+\tau_{0} ; r_{0}\right) e^{j 2 \pi \rho \tau}\right](u) \\
& \cdot \exp \left\{-j \frac{4 \pi\left(r_{0}+\Delta r_{v}\right)}{\lambda} \gamma\left(f_{a}\right)-j \Theta\right\} \\
& =C_{3} \sin c\left(u+\tau_{0} \cos \alpha-\rho \sin \alpha\right) \cdot \\
& \exp \left\{j \pi\left(\begin{array}{l}
\tau_{0}^{2} \sin \alpha \cos \alpha+2 u \tau_{0} \sin \alpha+2 u \rho \cos \alpha \\
-\rho^{2} \sin \alpha \cos \alpha-2 \rho \tau_{0} \sin \alpha
\end{array}\right)\right\} \\
& \cdot \exp \left\{-j \frac{4 \pi\left(r_{0}+\Delta r_{v}\right)}{\lambda} \gamma\left(f_{a}\right)-j \Theta\right\}
\end{aligned}
$$


Table 1. Simulation parameters.

\begin{tabular}{|c|c|c|c|}
\hline Parameter & Value & Parameter & Value \\
\hline \hline Carrier Wavelength & $0.24 \mathrm{~m}$ & Forward Velocity & $180 \mathrm{~m} / \mathrm{s}$ \\
\hline Bandwidth for Transmit Signal & $30 \mathrm{MHz}$ & Duration of Synthetic Aperture & $5.12 \mathrm{~s}$ \\
\hline Pulse Duration of Transmit Signal & $10 \mu \mathrm{s}$ & Pulse Repetition Frequency & $100 \mathrm{~Hz}$ \\
\hline Sampling Frequency in Range Direction & $40 \mathrm{MHz}$ & Range-independent Motion Errors & $t^{2}$ \\
\hline
\end{tabular}

In order to eliminate the influence of RCM term, it requires

$$
\begin{array}{r}
-\frac{2}{c} r_{r e f} C_{s} \cos \alpha-\rho \sin \alpha=0 \\
\Rightarrow \rho=-\frac{2}{c} r_{r e f} C_{s} \cot \alpha=\frac{4}{c} r_{r e f} k_{m} C_{s}\left(1+C_{s}\right) \\
\Rightarrow H_{r c m c}(\tau, \rho)=e^{j \frac{4 \pi}{c} r_{r e f} k_{m} C_{s}\left(1+C_{s}\right) \tau}
\end{array}
$$

The next step is to compensate to the residual phase, so the phase compensation function is:

$$
H_{v}\left(f_{a}, r_{0}\right)=\exp \left\{\begin{array}{l}
-j \pi\left(\begin{array}{l}
\tau_{0}^{2} \sin \alpha \cos \alpha+2 u \tau_{0} \sin \alpha+2 u \rho \cos \alpha \\
-\rho^{2} \sin \alpha \cos \alpha-2 \rho \tau_{0} \sin \alpha
\end{array}\right) \\
+j \Theta\left(f_{a}, r_{0}\right)
\end{array}\right\}
$$

In the following, second-order motion compensation in two-dimensional time-domain is performed, and the compensation function is:

$$
H_{\text {moco } 2}\left(t, r_{0}\right)=\exp \left\{-j \frac{4 \pi}{\lambda} \Delta r_{v}\left(t ; r_{0}\right)\right\}
$$

After this step, range-dependent motion errors can be removed.

The last step is to carry out azimuth-FrFT in rangeDoppler domain, so

$$
\begin{aligned}
& s d\left(t, u ; r_{0}\right)=\int_{-\infty}^{+\infty} S d_{2}\left(f_{a}, u ; r_{0}\right) K_{p}\left(f_{a}, t\right) d f_{a} \\
& =C_{4} \sin c\left(\tau-\frac{2 r_{0}}{c}\right) \int_{-\infty}^{+\infty} r e c t\left[\frac{f_{a}}{B_{d}}\right] \exp \left\{-j \frac{4 \pi r_{0}}{\lambda} \gamma\left(f_{a}\right)\right\} \\
& \cdot \exp \left\{j \pi\left(\left(f_{a}^{2}+t^{2}\right) \cot \beta-2 f_{a} t \csc \beta\right)\right\} d f_{a}
\end{aligned}
$$

where $\quad S d_{2}\left(f_{a}, u ; r_{0}\right)=C_{3} \sin c\left(u-\frac{2 r_{0}}{c}\right) \cdot \exp \left\{-j \frac{4 \pi r_{0}}{\lambda} \gamma\left(f_{a}\right)\right\}$, which is the signal in range-Doppler domain after secondorder motion compensation. $\gamma\left(f_{a}\right)=\sqrt{1-\left(\frac{\lambda f_{a}}{2 v}\right)^{2}} \approx 1-\frac{1}{2}\left(\frac{\lambda f_{a}}{2 v}\right)^{2}$.

$$
\text { Choosing } \cot \beta=-\frac{\lambda \pi r_{0}}{2 v^{2}}, p_{\text {opt }}=-\frac{2}{\pi} \operatorname{artcot}\left(\frac{\lambda \pi r_{0}}{2 v^{2}}\right) \text {, then }
$$

$$
\begin{aligned}
s d\left(t, u ; r_{0}\right)= & C_{4} \sin c\left(\tau-\frac{2 r_{0}}{c}\right) \exp \left\{-j \frac{4 \pi r_{0}}{\lambda}\right\} \exp \left\{-j \frac{\lambda \pi r_{0}}{2 v^{2}} t^{2}\right\} \\
& \quad \int_{-\infty}^{+\infty} r e c t\left[\frac{f_{a}}{B_{d}}\right] \cdot \exp \left\{-j \pi f_{a} t \csc \beta\right\} d f_{a} \\
= & C_{5} \sin c\left(\tau-\frac{2 r_{0}}{c}\right) \sin c(\csc \beta t)
\end{aligned}
$$

\section{SIMULATIONS AND SAR IMAGING RESULTS BASED ON REAL DATA FOR THE PROPOSED AL- GORITHM}

\subsection{Simulations of Point Target for the Proposed Algo- rithm}

Simulations of point target with motion error for the algorithm put forward in this paper are as follows; the simulation parameters are shown in Table $\mathbf{1}$.

The range motion error is non-stationary random motion error whose mean value is exponential function, so rangedependent motion error is $\Delta r_{v}\left(t, r_{0}\right)=\Delta r\left(t, r_{0}\right)-\Delta r_{m}\left(t, r_{m}\right)$.

Fig. (2) is a simulation comparison of the conventional CS algorithm based on FFT and the improved CS algorithm based on FrFT and motion compensation about point target with motion error.

From the simulation results, it is known that both of the algorithms can eliminate the influence of motion error effectively. The azimuth impulse responses after compensation are close to that when there is no motion error. Fig. (2c and d) indicates that the main-lobes of impulse response both in the range and azimuth direction after processing using the proposed algorithm in this paper, are narrowed than that taking the conventional FFT-based CS algorithm, which proves that the new algorithm can improve the resolution and obtain good focusing effect.

At the same time, picture (2c) also shows that the first side-lobe of range impulse response for the proposed algorithm falls below $-35 \mathrm{~dB}$, which for the conventional algorithm only reaches about $-25 \mathrm{~dB}$ and there is a burr in $-11 \mathrm{~dB}$, all of which proves that the effect of suppression side-lobe and anti-interference for the proposed algorithm is obvious. The performance comparison of the two algorithms is shown in Table $\mathbf{2}$ and Table $\mathbf{3}$. 


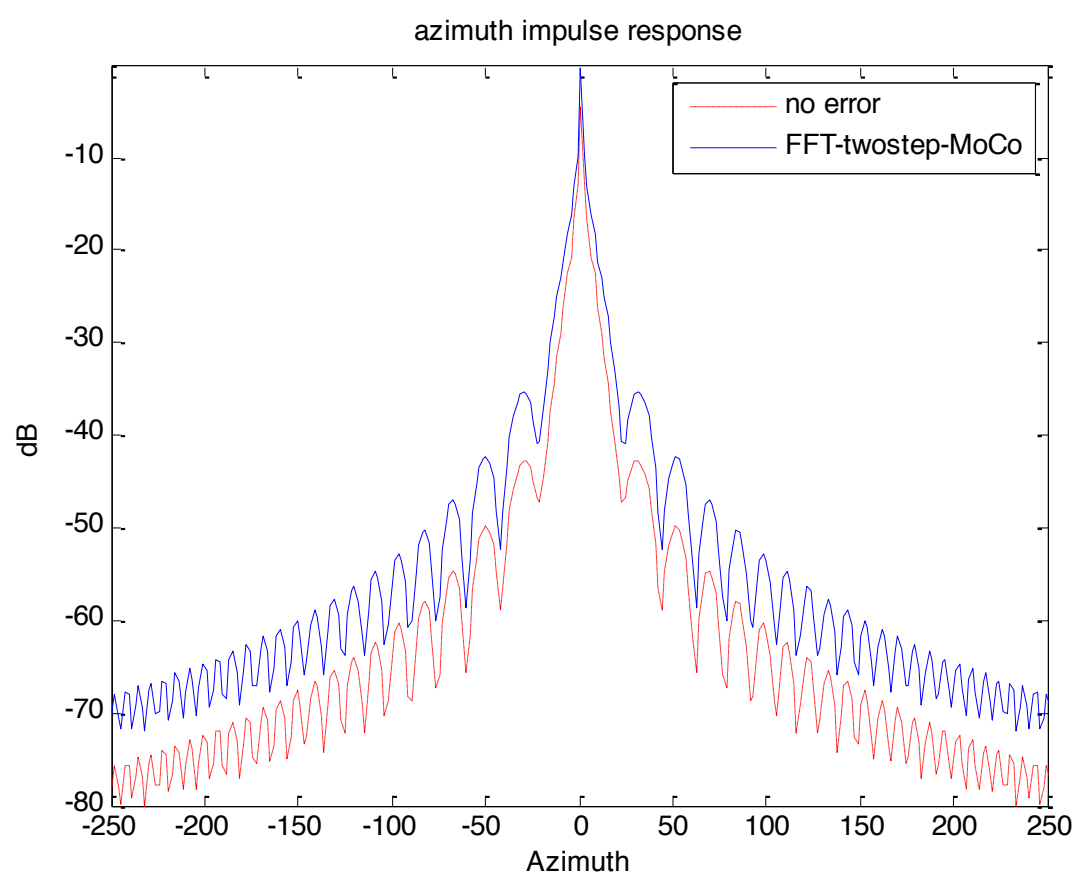

(a) Comparison of azimuth impulse response when no error and when error exists but processed taking the conventional motion compensation algorithm

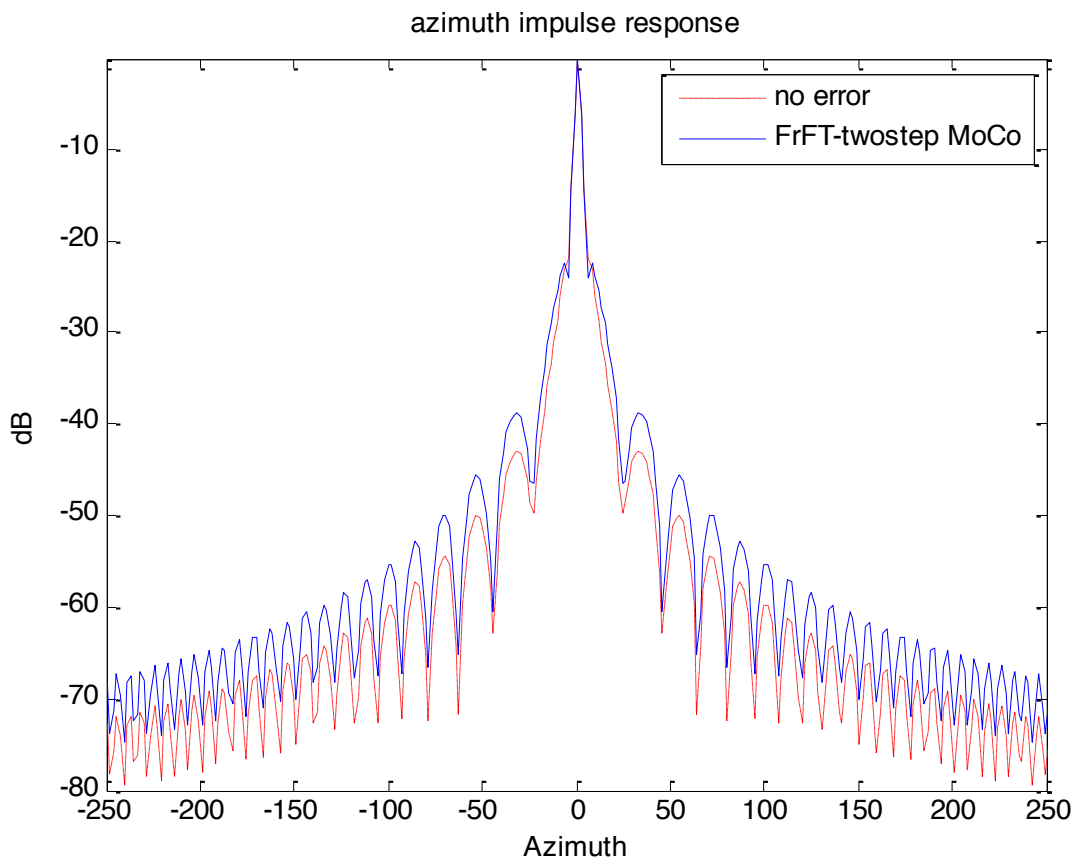

(b) Comparison of azimuth impulse response when no error and when error exists but processed using the proposed compensation algorithm

Fig. (2). Contd... 


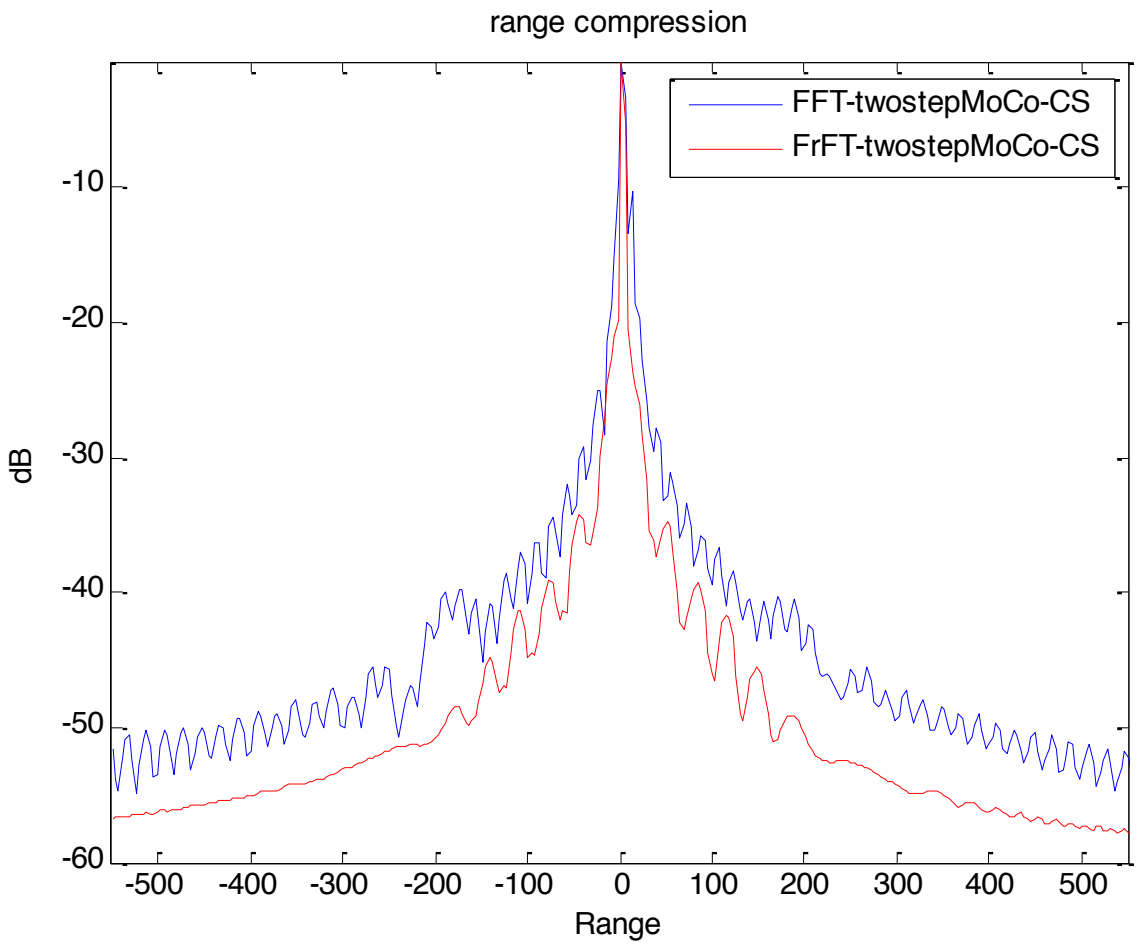

(c) Comparison of range impulse response after the two compensation algorithms processed

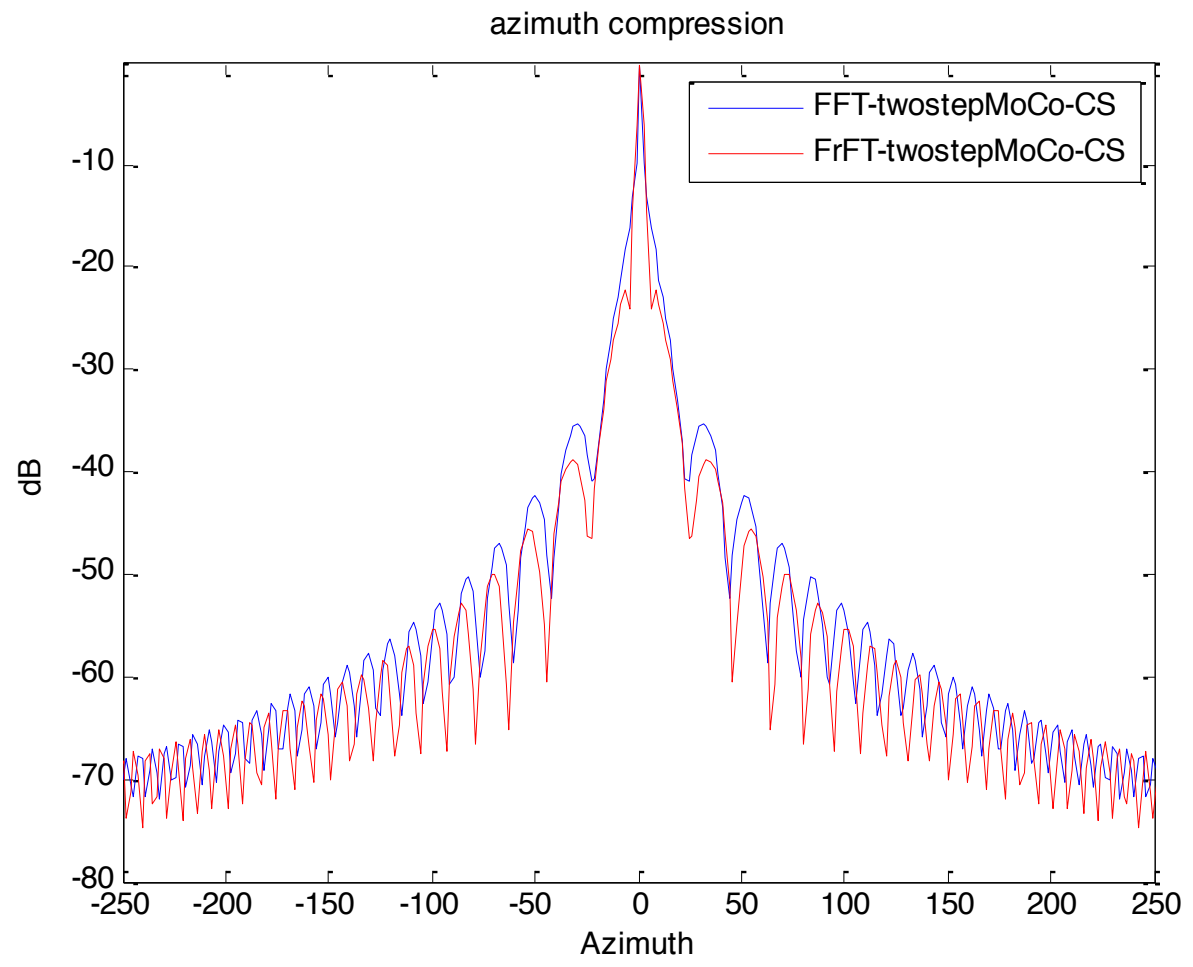

(d) Comparison of azimuth impulse response after the two compensation algorithms processed

Fig. (2). Comparison of point target simulations for the conventional and the proposed algorithm. 
Table 2. Performance comparison of azimuth impulse response.

\begin{tabular}{|c|c|c|c|}
\hline & Main Lobe & ISLR & PSLR \\
\hline \hline No Error & $1.05 \mathrm{~m}$ & $-24.311 \mathrm{~dB}$ & $-32.012 \mathrm{~dB}$ \\
\hline FFT-two-step Motion Compensation CSA & $1.0906 \mathrm{~m}$ & $-15.556 \mathrm{~dB}$ & $-21.038 \mathrm{~dB}$ \\
\hline FrFT- two-step Motion Compensation CSA & $1.0825 \mathrm{~m}$ & $-23.109 \mathrm{~dB}$ & $-31.332 \mathrm{~dB}$ \\
\hline
\end{tabular}

Table 3. Performance comparison of range impulse response.

\begin{tabular}{|c|c|c|}
\hline & Main Lobe & ISLR \\
\hline \hline No Error & $1.195 \mathrm{~m}$ & $-25.361 \mathrm{~dB}$ \\
\hline FFT- two-step Motion Compensation CSA & $1.1416 \mathrm{~m}$ & $-15.884 \mathrm{~dB}$ \\
\hline FrFT- two-step Motion Compensation CSA & $1.209 \mathrm{~m}$ & $-24.110 \mathrm{~dB}$ \\
\hline
\end{tabular}

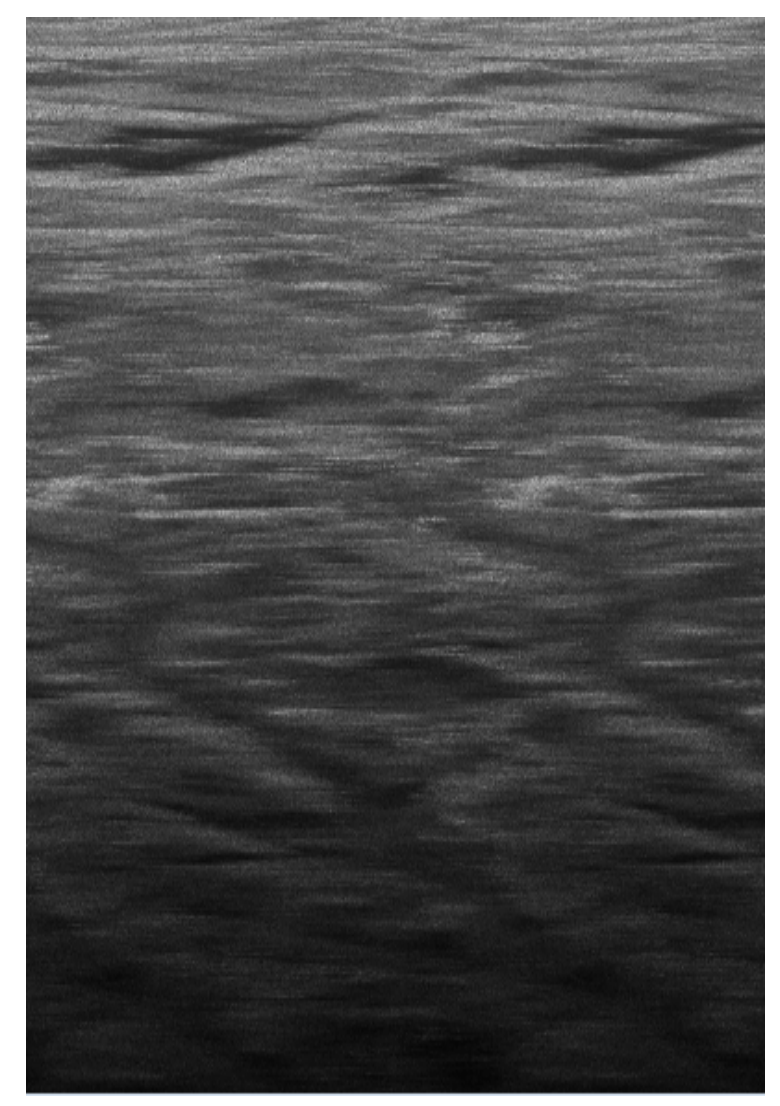

(a) SAR image with motion error

Fig. (3). Contd... 


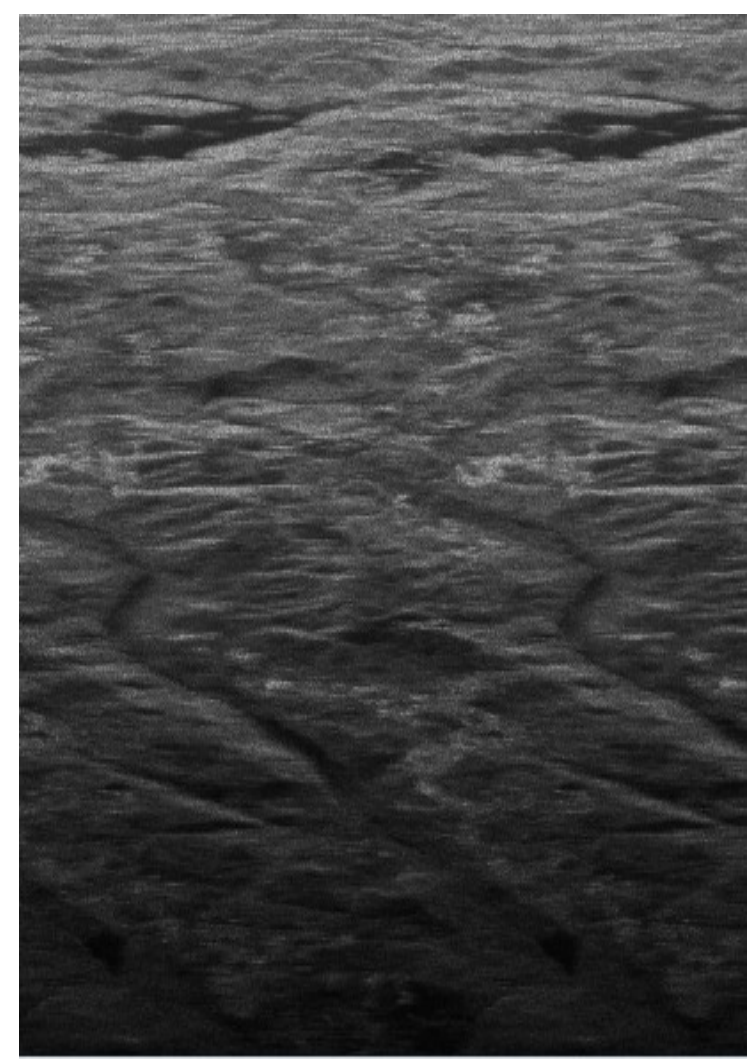

(b) SAR image after FFT-two step motion compensation CSA

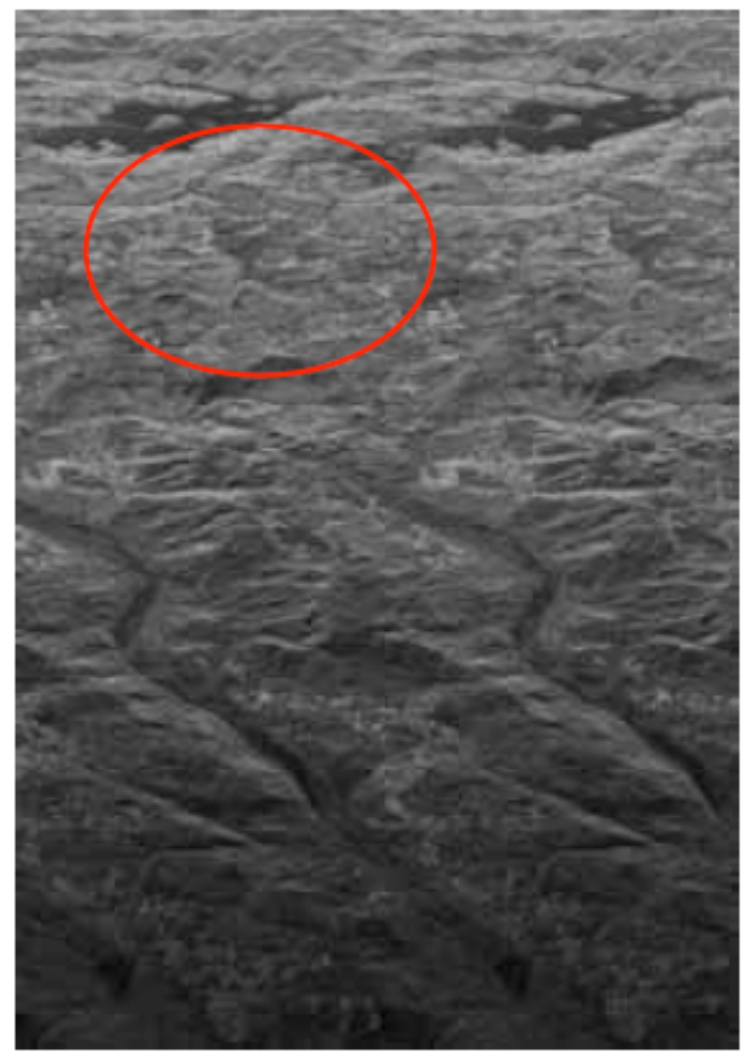

(c) SAR image after FrFT-two step motion compensation CSA

Fig. (3). Comparison of imaging results before and after motion compensation (resolution: $3 m \times 3 m$ ). 


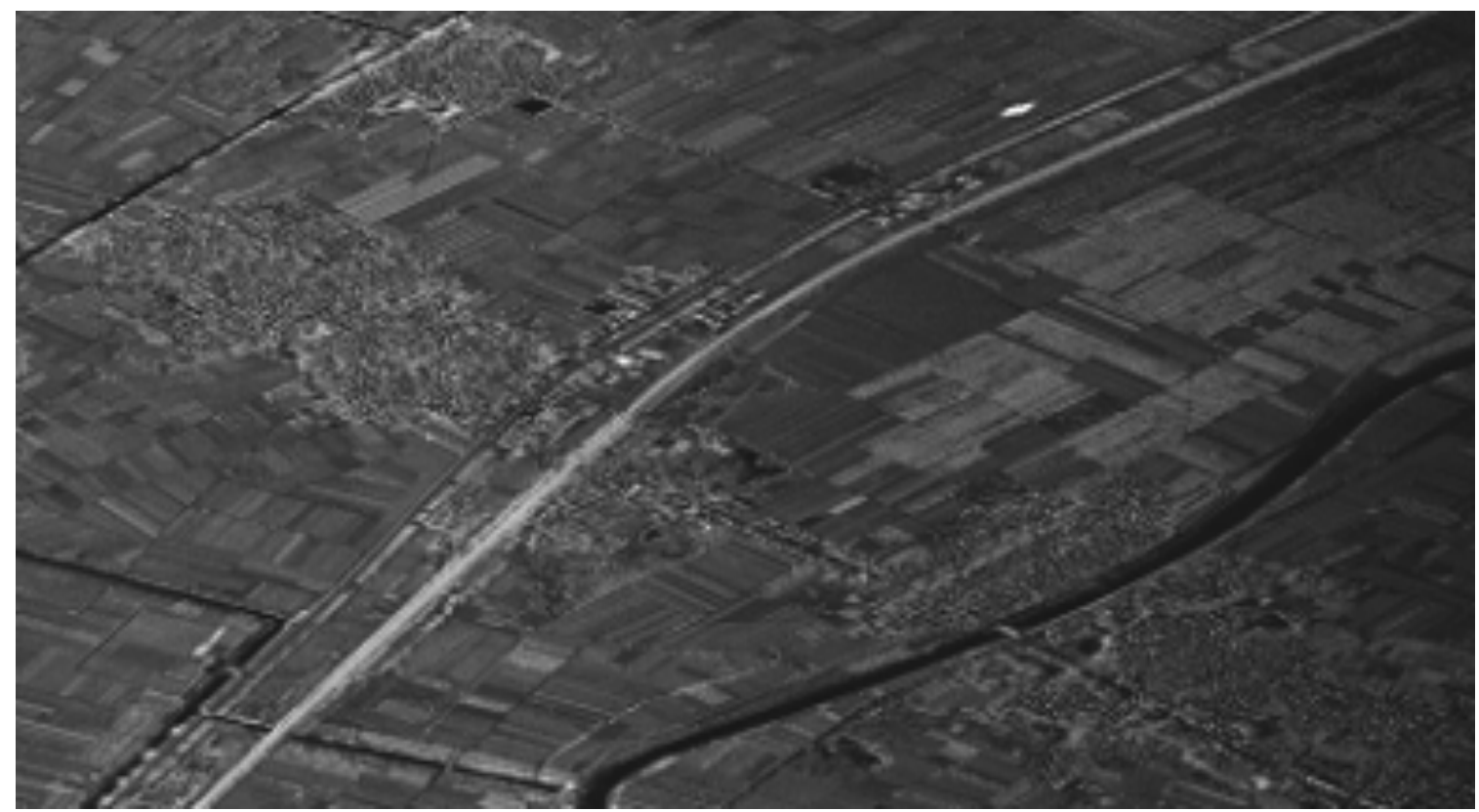

(a) SAR image after FFT-two-step motion compensation CS algorithm processing

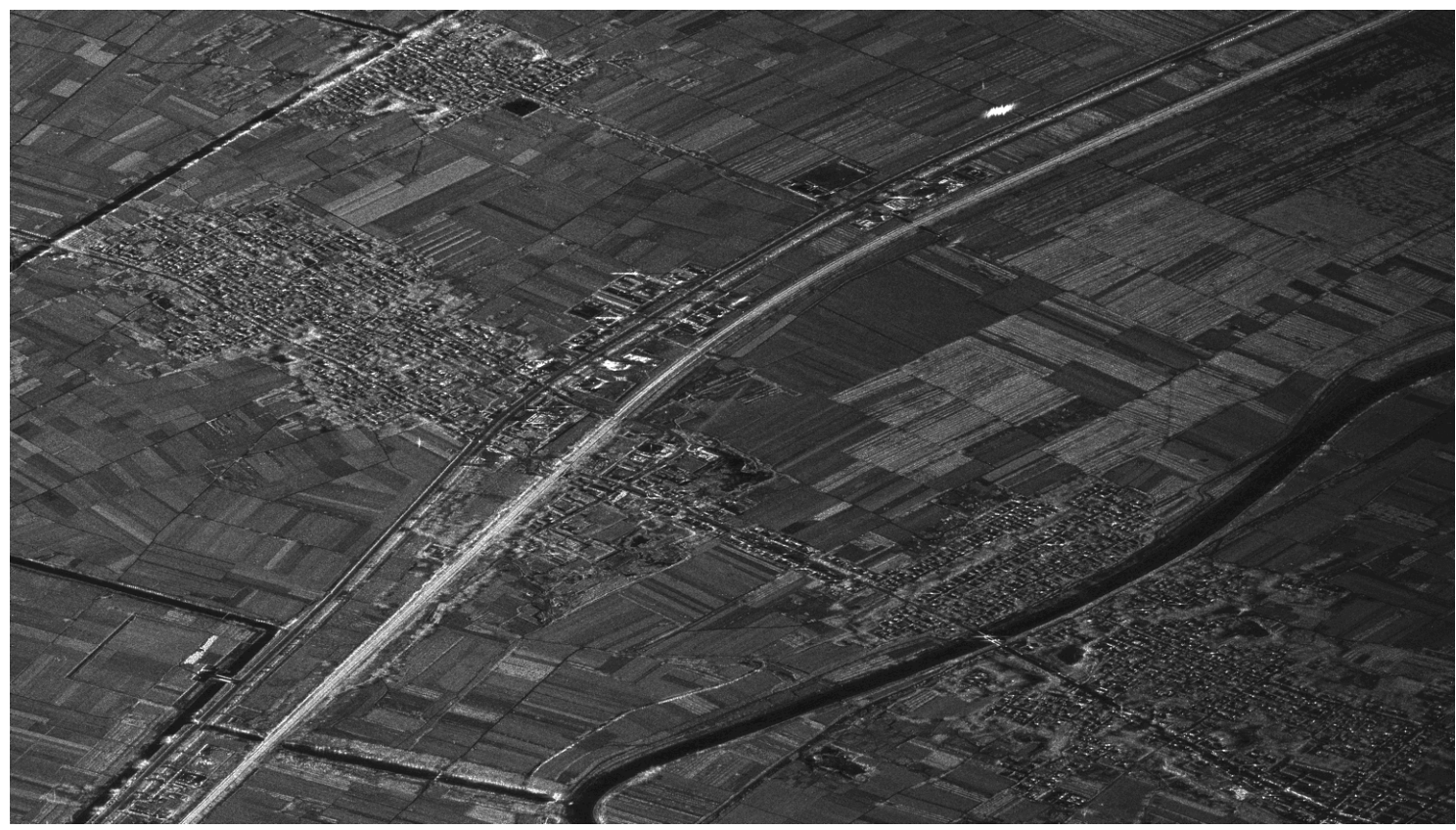

(b) SAR image after FrFT- two-step motion compensation CS algorithm processing

Fig. (4). Imaging results of X-SAR data combined motion compensation (resolution: $0.5 m \times 0.5 m$ ).

\subsection{Comparison of Imaging Results of Real L-SAR Data}

Fig. (3a) is imaging result of the real SAR data with motion errors. The blurring image shows there are obvious quadratic phase errors.

Processing result for such SAR data using the conventional CS algorithm based on FFT and two-step motion compensation is shown as Fig. (3b). Due to the elimination of most motion errors, image resolution is improved significantly, but some areas are with more details (such as parts of the circle line), the image is not clear and image resolution deteriorates because of the residual phase error.
The processing result with the improved CS imaging algorithm based on FrFT and two-step motion compensation technology is shown in Fig. (3c). As the detailed information increases, the image resolution is further improved.

\subsection{Comparison of Imaging Results of Real X-SAR Data}

Fig. (4) shows imaging results of X-SAR data. Since the motion error is stable and the azimuth beam is narrow, so the processing results using the conventional algorithm and using the proposed algorithm are approximately the same. But in some areas with more details, Fig. (4b) is clearer than Fig. 
(4a), which further proves that the proposed CS algorithm based on FrFT and motion compensation can effectively enhance the resolution of SAR images.

\section{CONCLUSION}

Motion error is a crucial factor to limit airborne SAR resolution improvement. Due to the continuous pursuit of modern SAR for high resolution, how to overcome the motion error caused by air turbulence becomes an urgent problem for radar workers. The improved CS imaging algorithm based on fractional Fourier transform and two-step motion compensation proposed in this paper can reduce various motion errors effectively, especially it can eliminate the image blurring caused by non-stationary motion errors. The research results of the paper provide an effective solution scheme to motion error processing.

\section{CONFLICT OF INTEREST}

The authors confirm that this article content has no conflict of interest.

\section{ACKNOWLEDGEMENTS}

This paper is supported by the natural science foundation of Fujian province of 2013 project (the number is 2013J01242).

\section{REFERENCES}

[1] Y.Li, Xing-dong Liang, C. Ding, L. Zhou, L. Chen, and W. Hong, "A motion compensation approach integrated in the omega-k algorithm for airborne SAR", IEEE International Conference on Imaging Systems and Techniques, Manchester, United Kingdom, pp. 245-248, 2012.
[2] Y. Mao, M. Xiang, L. Wei, Y. Li, and W. Hong, "Error analysis of SAR motion compensation", IEEE International Conference on Imaging Systems and Techniques, Manchester, United Kingdom, 2012, pp. 377-380.

[3] H. Guo, and Y. Li, "Studying atmospheric turbulence effects on aircraft motion for airborne SAR motion compensation requirements", IEEE International Conference on Imaging Systems and Techniques, Manchester, United Kingdom, 2012, pp. 152-157.

[4] V. Namias, "The fractional Fourier transform and its application in quantum mechanics", Order of IMA Journal of Applied Mathematics, vol. 25, pp. 241-265, 1980.

[5] Q. Lin, T. Ran, Z. Siyong, and W. Yue, "Adaptive time-varying filter for linear FM signal in fractional Fourier domain", $6^{\text {th }} I E E E$ International Conference on Signal Processing, Beijing, China, pp.1425-1428, 2002

[6] L. Durak, and S. Aldirmaz, "Adaptive fractional Fourier domain filtering”, Signal Processing, vol. 90. no. 4, pp. 1188-1196, 2010.

[7] S. E. D. Igor, and S. L. Jubia, "Fractional Fourier transform as a signal processing tool: an overview of recent developments", Signal Processing, vol. 91, no. 6, pp. 1351-1369, 2011.

[8] A. S. Amein, "A new chirp scaling algorithm based on the fractional Fourier transform”, IEEE Signal Processing Letters, vol. 12, no. 10, pp. $705-708,2005$.

[9] S. Ahmed, J.J. Amein, and J.J. Soraghan, “Azimuth fractional transformation of the fractional chirp scaling algorithm", IEEE Transactions on Geosciences and Remote Sensing, vol. 44, no. 10, pp. 2871-2879, 2006.

[10] C. Clemente, and J. J. Soraghan, "Range Doppler and chirp scaling processing of synthetic aperture radar data using the fractional Fourier transform", IET Signal Processing, vol. 6, no. 5, pp. 503-510, 2012.

[11] M.G. El-Mashed., and M.I. Dessouky, "Target image enhancement in radar imaging using fractional Fourier transform", Sense Imaging, vol. 13, pp. 37-53, 2012.

[12] R. Lanari, and G. Fornaro, "A short discussion on the exact compensation of the SAR range-dependent range cell migration effect", IEEE Transactions on Geoscience and Remote Sensing, vol. 35, pp. 1446-1452, 1997.

[13] T. Ge-wei, and D. Yun-kai, "The two-dimensional spatial-variant properties of airborne SAR motion error and its compensation", Journal of Electronics and Information Technology, vol. 31, no. 2, pp. 366-369, 2009. 